\title{
Novel Disassembly Mechanisms of Sigmoid A $\beta 42$ Protofibrils by Introduced Neutral and Charged Drug Molecules
}

\author{
Xiaofeng Xing ${ }^{\dagger}$, Chengqiang $\mathrm{Liu}^{\dagger}$, Aqsa Ali ${ }^{\dagger}$, Baotao Kang ${ }^{\dagger}$, Ping $\mathrm{Li}^{\S}{ }^{\xi}$, ,Hongqi Ai, ${ }^{\dagger}{ }^{*}$ \\ ${ }^{\dagger}$ School of Chemistry and Chemical Engineering, University of Jinan, Jinan 250022, PR China \\ ${ }^{\S}$ Key Laboratory of Life-Organic Analysis, School of Chemistry and Chemical Engineering, Qufu \\ Normal University, Qufu, 273165, China
}

*To whom correspondence should be addressed, E-mail:chm_aihq@ujn.edu.cn; lignip@163.com

\section{Contents}

Figures S1-S2

Figures S3-S4

Figures S5-S6

Figures S7-S8

Figure S9

Figure S10

Figure S11

Table S1

Table S2

Tables S3-S4

Table S5
Page2

Page3

Page4

Page5

Page6

Page7

Page8

Page9

Page9 - Page10

Page10

Page10 - Page15 

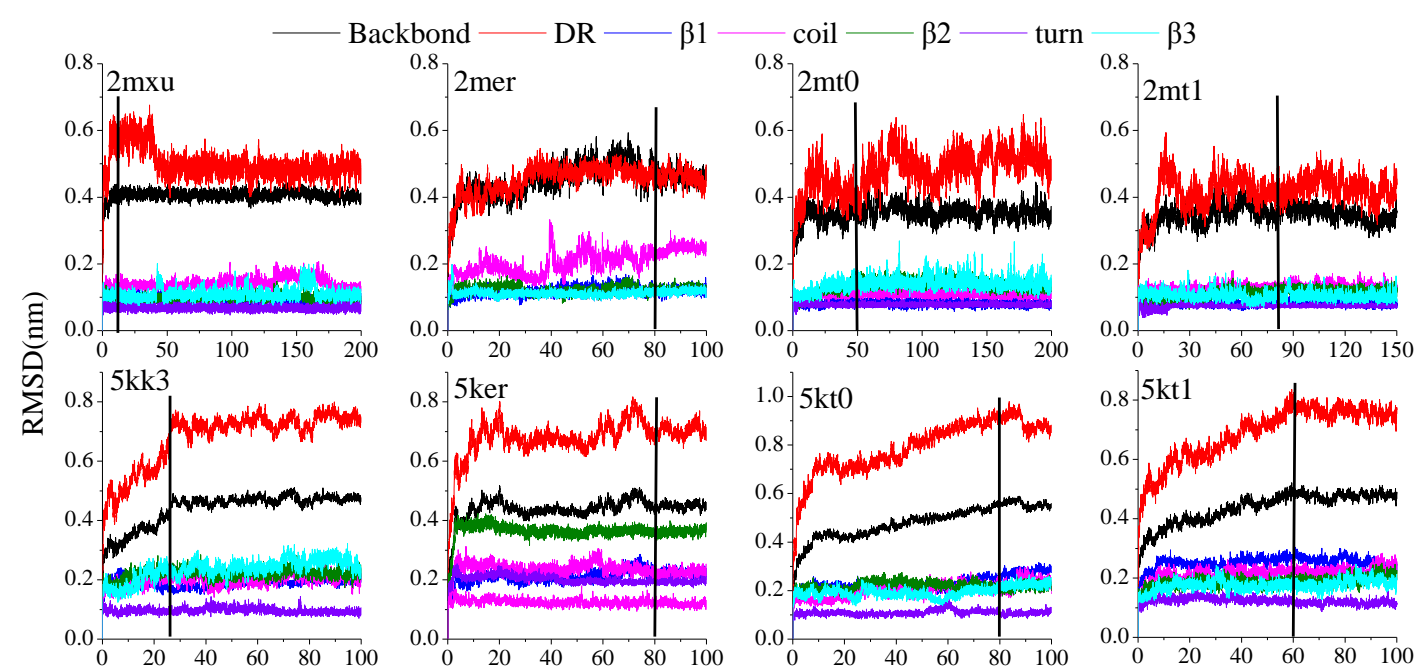

(a) Time(ns)

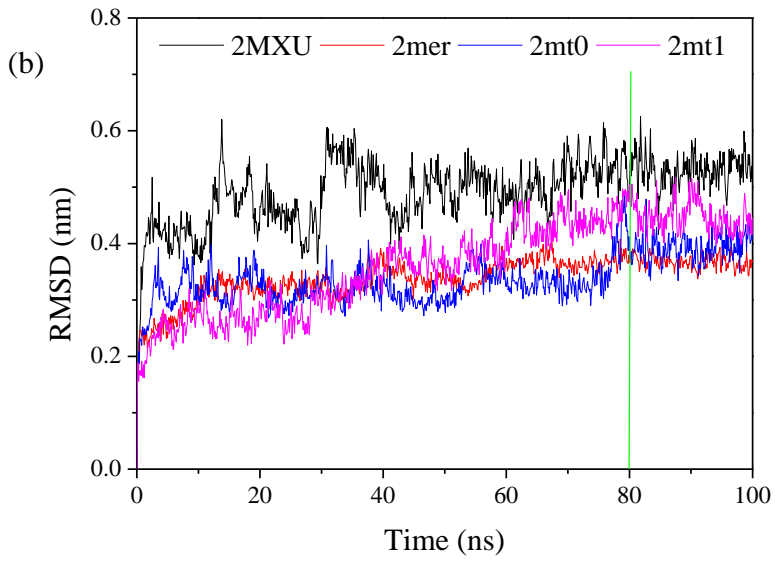

Figure S1 (a) RMSDs of two S-shaped A $\beta 42$ fibrils 2MXU and 5KK3 and their complexes 2mer, $2 \mathrm{mt} 0,2 \mathrm{mt} 1,5 \mathrm{ker}, 5 \mathrm{kt} 0,5 \mathrm{kt} 1$ obtained by employing gromos $53 \mathrm{a} 6$ force field, in which the black represents total RMSD, whereas the red, the blue, the megenta, the olive, the navy, and the violet stand for the RMSDs of DR region, $\beta 1$ region, coil region, $\beta 2$ region, turn region, and $\beta 3$ region, respectively. (b) RMSDs of $2 \mathrm{mer}, 2 \mathrm{mt} 0,2 \mathrm{mt} 1$ obtained by employing Charmm $36 \mathrm{~m}$ force field. The periods after black vertical lines mark the equilibrium periods.

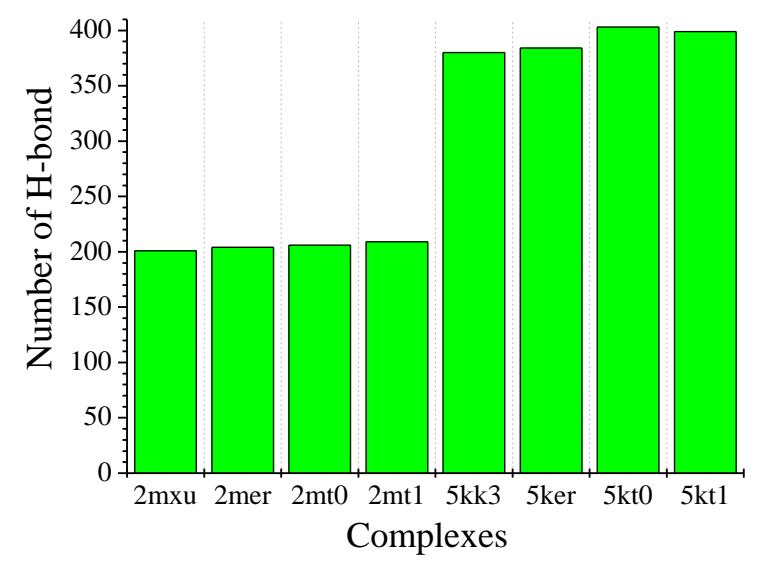

Figure S2. Number of H-bond in various complexes. 


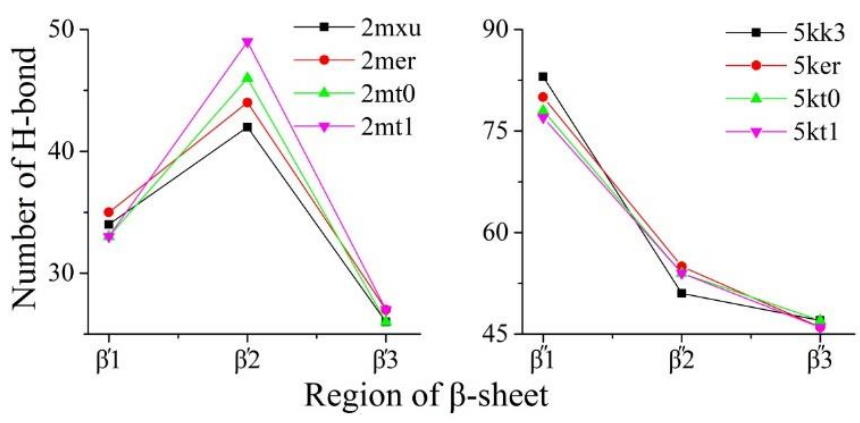

Figure S3. Number of H-bond in different $\beta$-sheet regions

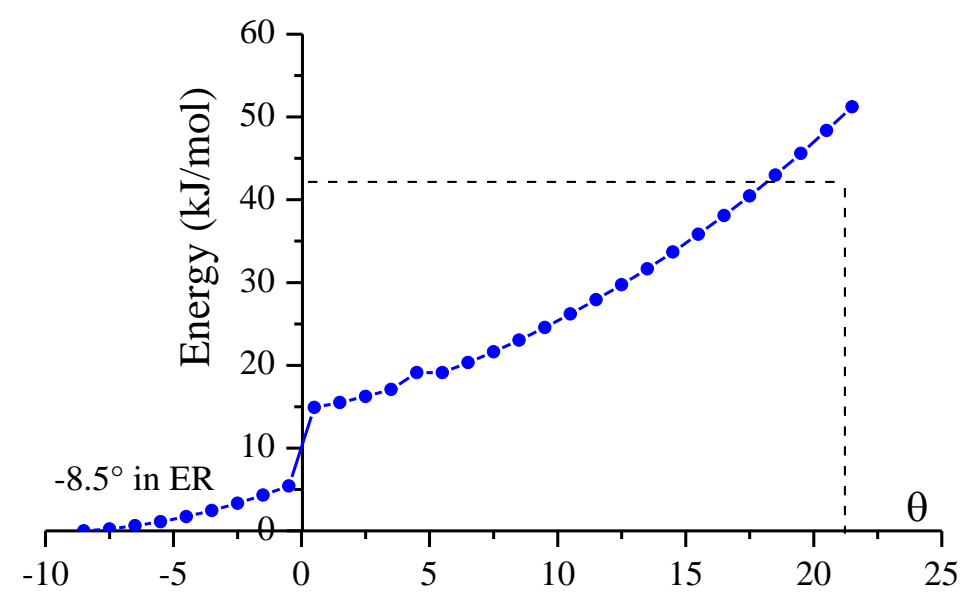

Figure S4. Energy changes of ER45 as the $\theta$ increase from $-8.5^{\circ}$ to $21.5^{\circ}$ in $2 \mathrm{mer}$. 

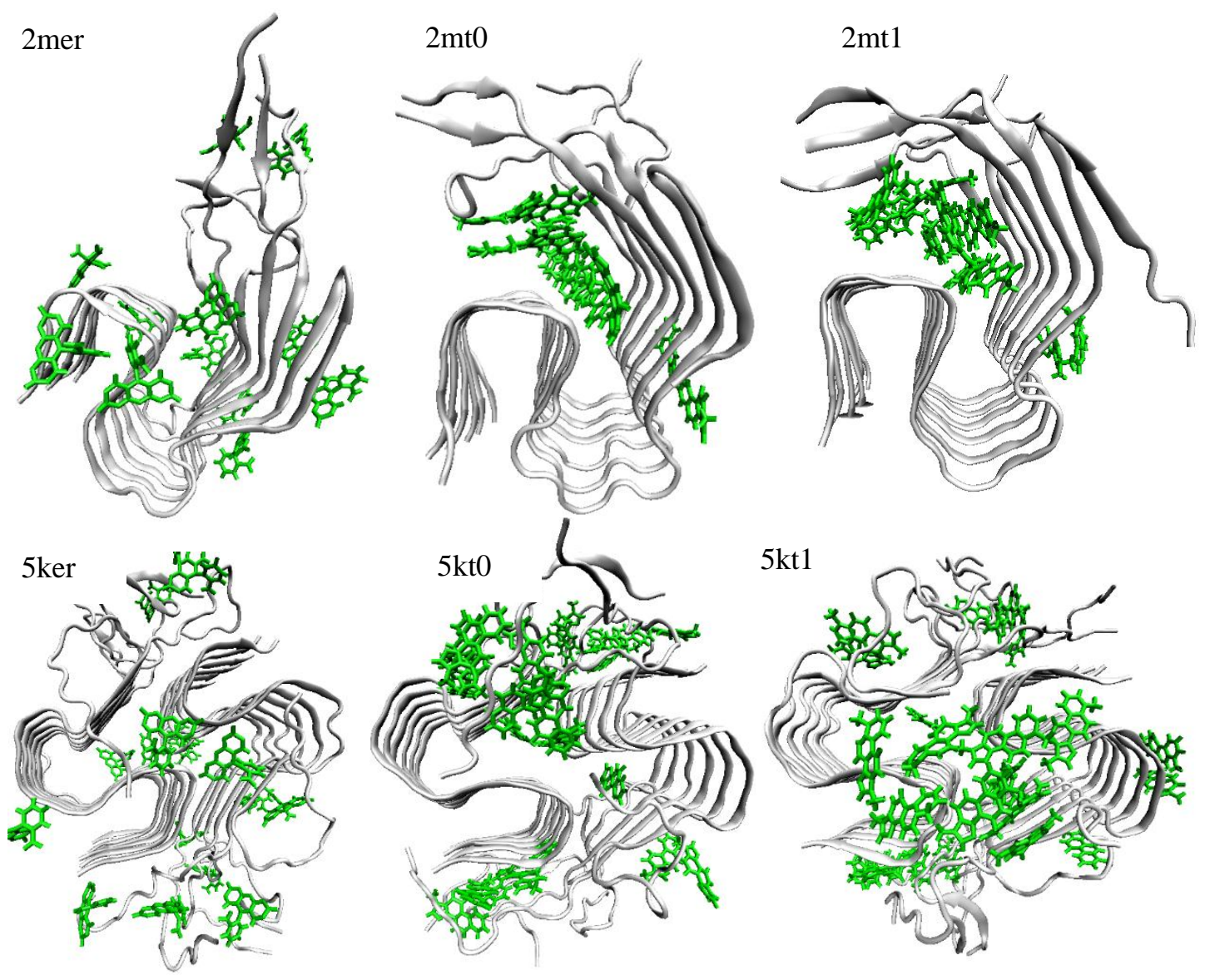

Figure S5. Distribution of drugs in $2 \mathrm{mer} / 2 \mathrm{mt} / 2 \mathrm{mt} 1 / 5 \mathrm{ker} / 5 \mathrm{kt} 0 / 5 \mathrm{kt} 1$ after MD.

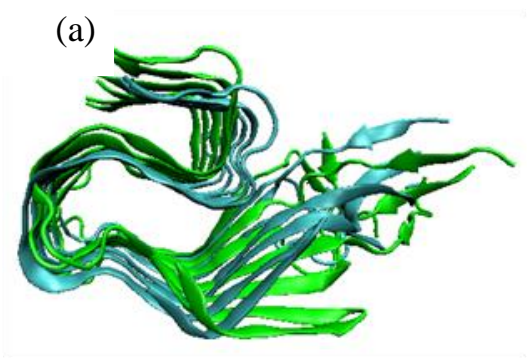

(d)

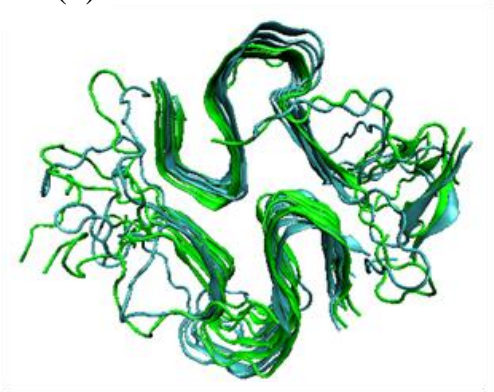

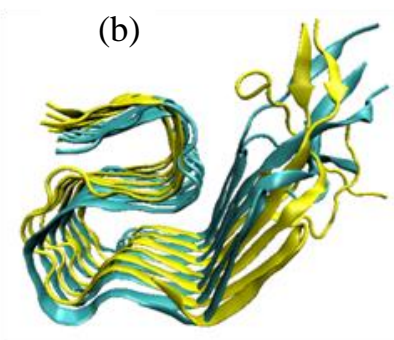

(e)

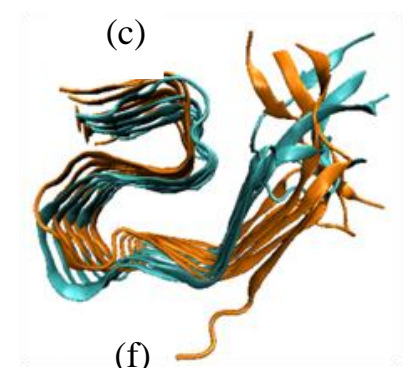

(f)

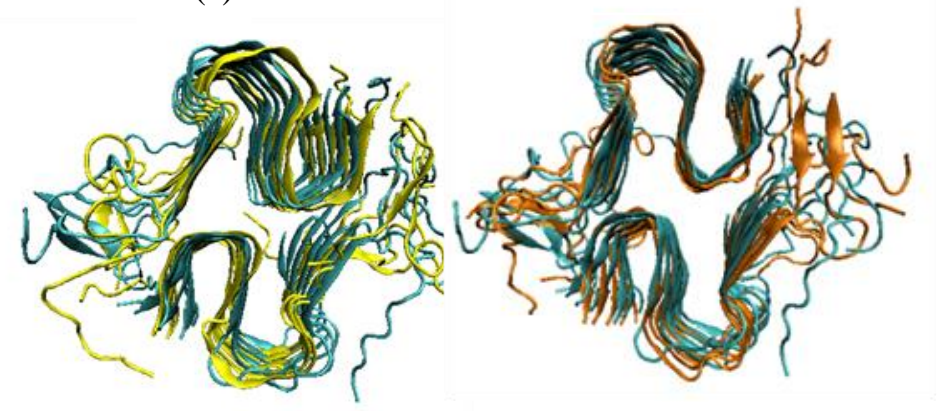

Figure S6. Least-squares fitted drug-involved structures on free 2MXU/5KK3. The free $2 \mathrm{MXU}$ and $5 \mathrm{KK} 3$ are shown in blue, whereas their drug-involved complexes are shown in different colors to distinguish them. (a) 2mer-green; (b) 2mt0-yellow; (c) 2mt1-orange; (d) 5ker-green; (e) 5kt0-yellow; (f) 5kt1-orange. 


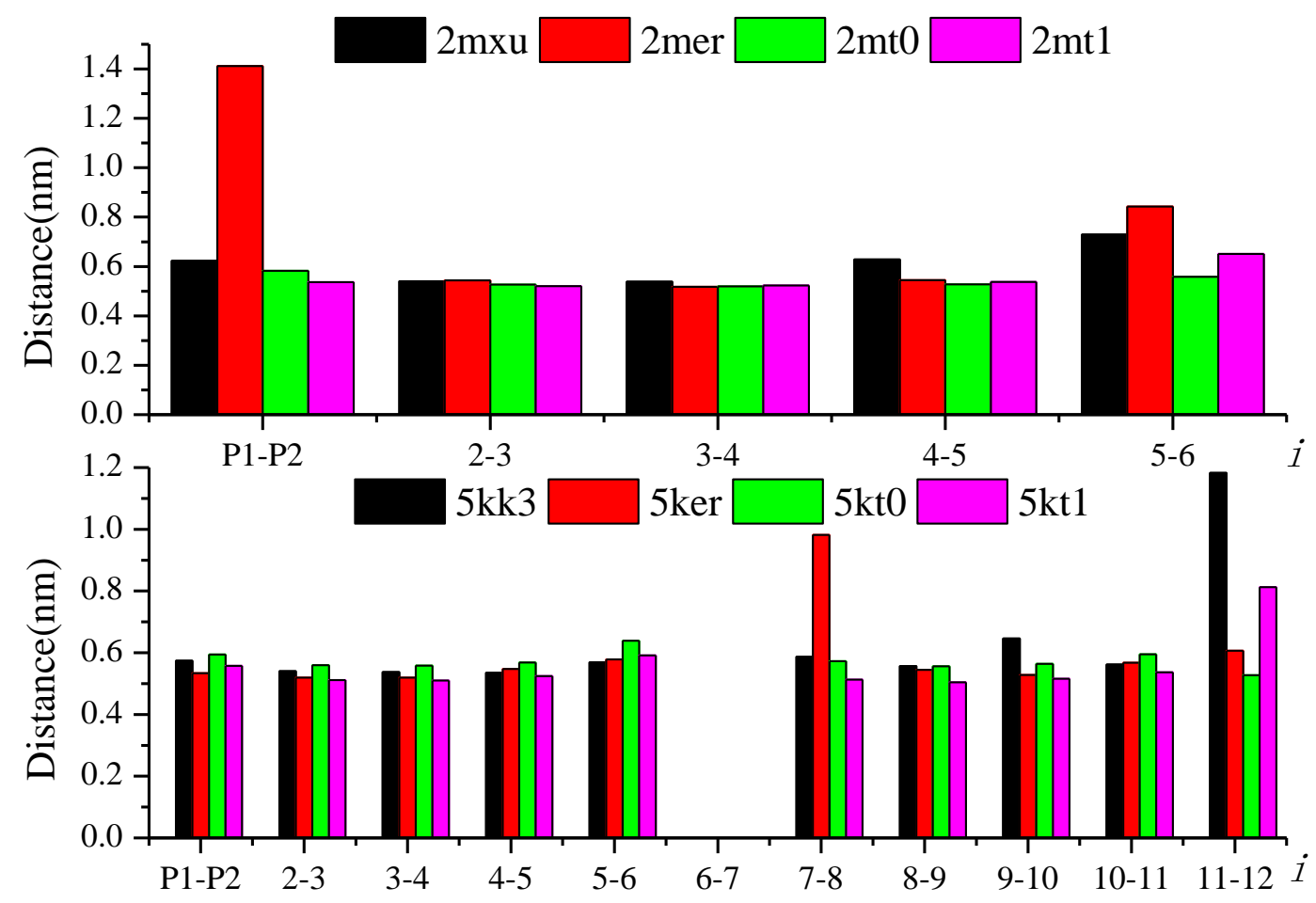

Figure $\mathrm{S} 7$ Interpeptide distance between adjacent peptides (Pi), measured by employing the $\mathrm{C} \alpha$ atom of Asp23 of each peptide.

( a )
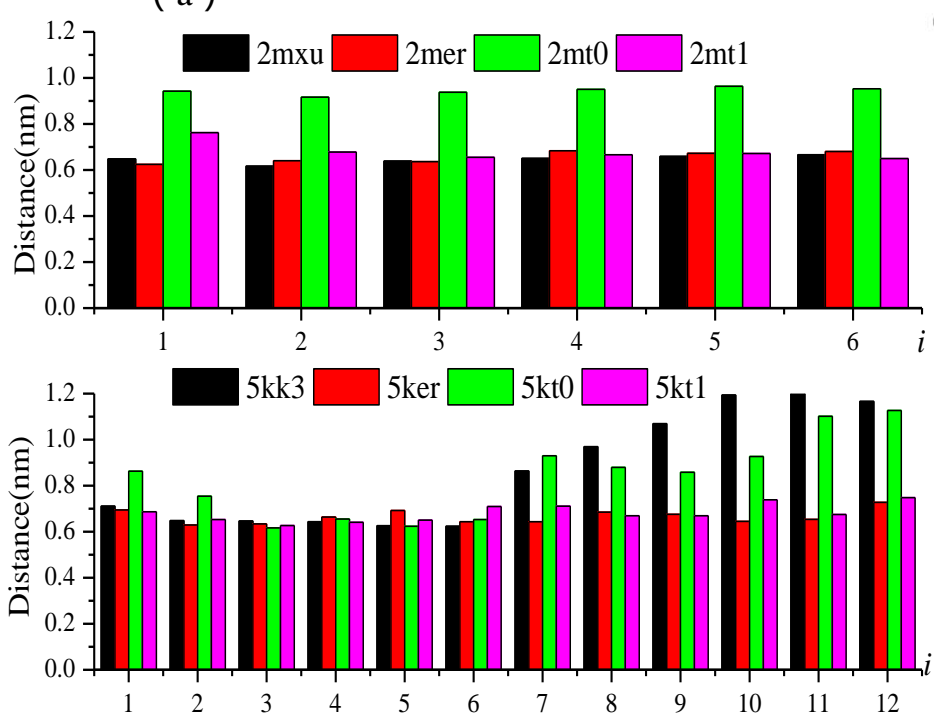

( b )
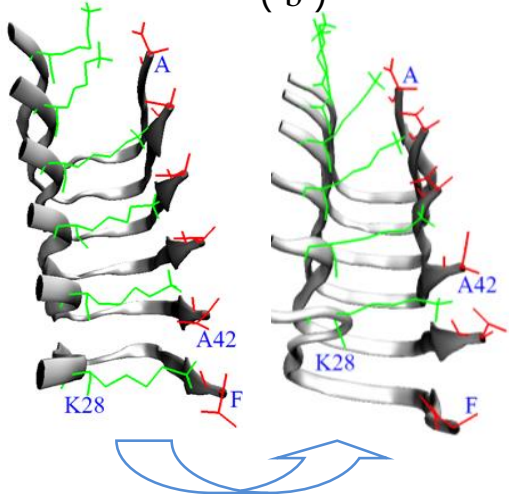

Figure S8. (a) Salt bridge distance measured by employing $\mathrm{C}_{\mathrm{G}}$ atom of $\mathrm{K} 28$ and $\mathrm{N}$ of A42. (b) the salt bridge of $2 \mathrm{mt} 0$ is twisted and the intramolecular salt bridge becomes intermolecular one. 

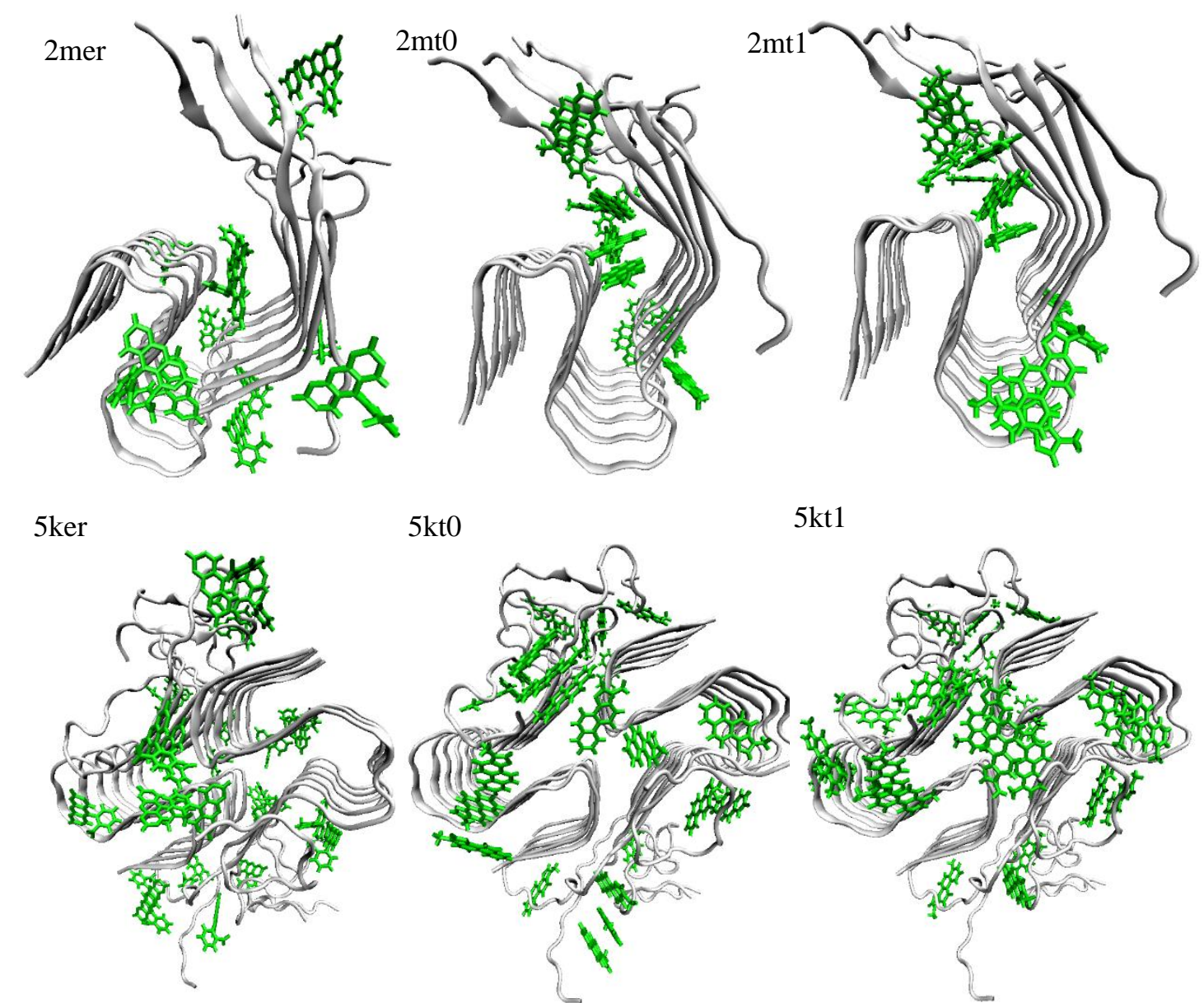

$5 \mathrm{kt} 1$

Figure S9. The initial distribution of drugs in $2 \mathrm{mer} / 2 \mathrm{mt} 0 / 2 \mathrm{mt} 1 / 5 \mathrm{ker} / 5 \mathrm{kt} 0 / 5 \mathrm{kt} 1$ by docking. 

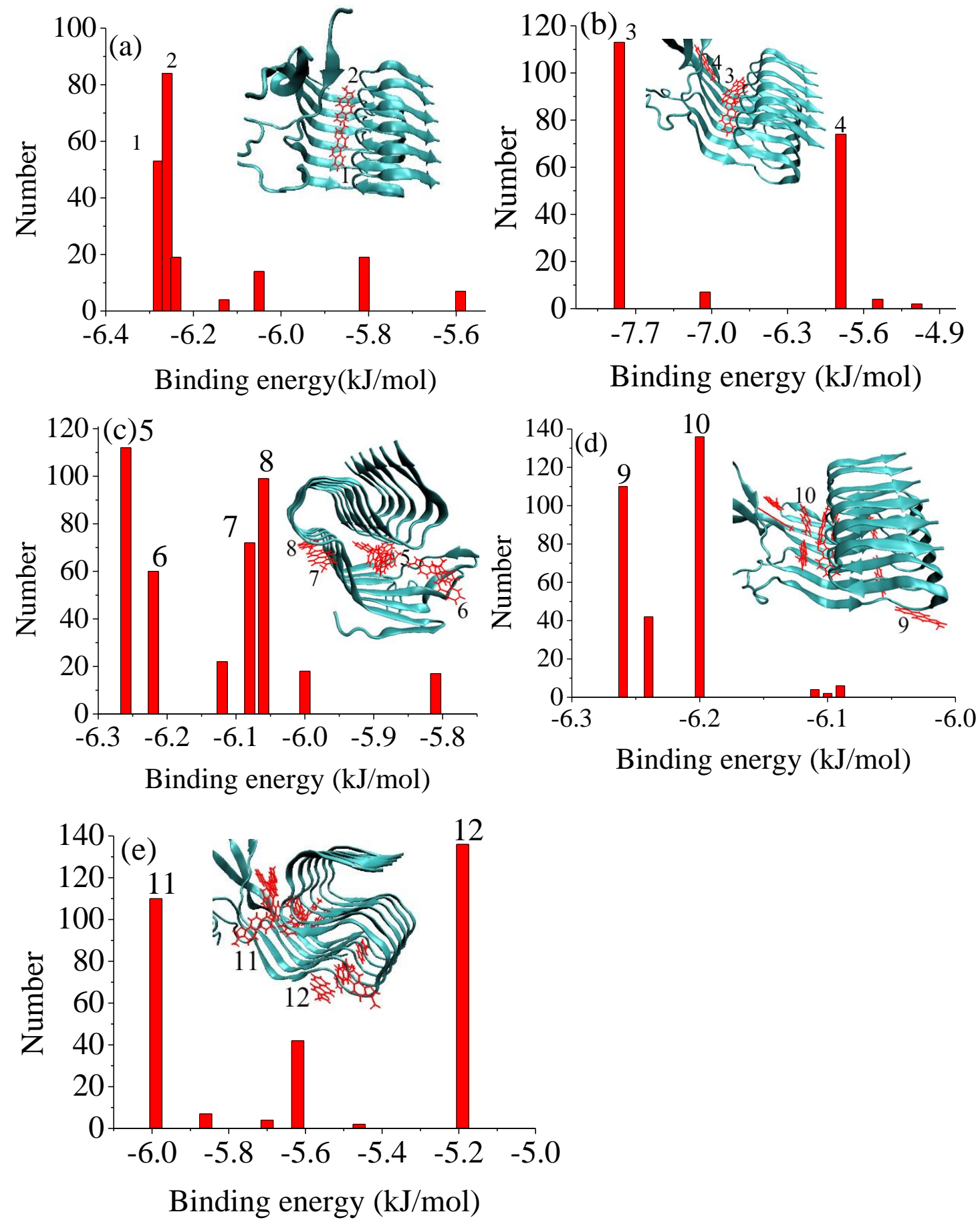

Figure S10. Alphabets of (a)-(e) show 5-step docking experiments for 2mt0 system, in which 12 TS0 molecules are docked to $2 \mathrm{MXU}$ progressively and the corresponding conformation number and binding free energy are displayed. 


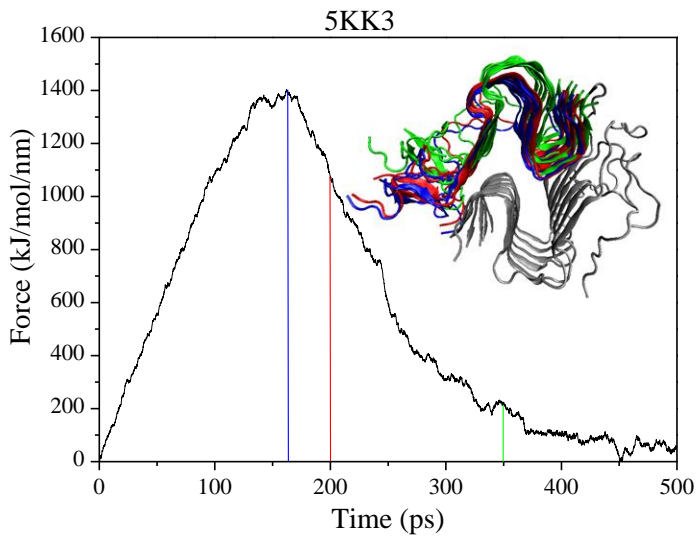

(a)

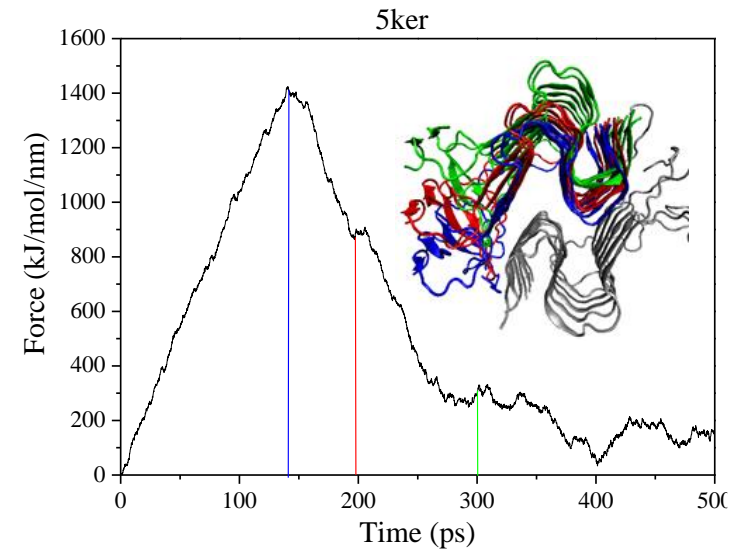

(b)

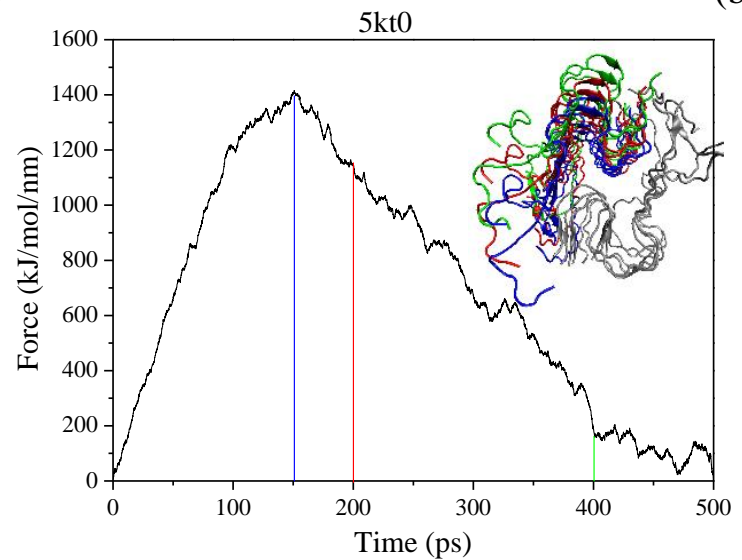

(c)

Figure S11. Dissociation pathways of A $\beta 42$ fibril, with corresponding plots of force vs time: (a) $5 \mathrm{KK} 3$, (b) $5 \mathrm{ker}$, (c) $5 \mathrm{kt} 0$. In each panel, the blue line (and structure) corresponds to the point of maximum force, and the red and green lines and structures correspond to major structural transitions $(5 \mathrm{KK} 3,5 \mathrm{ker}$, and $5 \mathrm{kt} 0)$ or the dissociation peptide set $\mathrm{A}$ (colored). 
Table S1. Binding energy ( $\mathrm{kJ} / \mathrm{mol}, 1^{\text {st }}$ row) of each region contributed, and the corresponding residues ( $2^{\text {nd }}$ row) and the drug number ( $3^{\text {rd }}$ row) in the region. The drug number denotes those within $10 \AA ̊$ of $2 \mathrm{MXU} / 5 \mathrm{KK} 3$.

\begin{tabular}{|c|c|c|c|c|c|c|c|}
\hline & $\mathbf{D R}^{\prime \prime \prime \prime}$ & $\beta 1^{\prime \prime \prime \prime}$ & & $\mathbf{L 1}^{\prime \prime \prime \prime}$ & $\beta 2^{\prime \prime \prime \prime}$ & $\mathbf{L 2}^{\prime \prime \prime \prime}$ & $\beta^{\prime \prime \prime \prime \prime}$ \\
\hline \multirow{3}{*}{ 2mer } & 290.7 & \multicolumn{2}{|c|}{-126.1} & 261.6 & -131.1 & -3.6 & 111.6 \\
\hline & E3/R5/D7/E11 & \multicolumn{2}{|c|}{ K16 } & E22/D23 & K28 & & A42 \\
\hline & 2 & \multicolumn{2}{|c|}{5} & 1 & 1 & 1 & 2 \\
\hline \multirow{3}{*}{$2 \mathrm{mt0}$} & -9.9 & \multicolumn{2}{|c|}{-1.8} & -5.5 & -7.6 & -15.7 & -5.6 \\
\hline & R5 & \multicolumn{2}{|c|}{ V12/K16/V18 } & & & L34 & \\
\hline & 5 & & & 2 & 0 & 0 & 0 \\
\hline \multirow{3}{*}{$2 \mathrm{mt1}$} & -13.8 & \multicolumn{2}{|c|}{-6.8} & -7.6 & -7.8 & -18.3 & -1.3 \\
\hline & R5 & \multirow{2}{*}{\multicolumn{2}{|c|}{$\begin{array}{c}\text { V12/K16/L17/V18 } \\
6\end{array}$}} & & & L34 & \\
\hline & 4 & & & 2 & 0 & 0 & 0 \\
\hline \multirow{3}{*}{ 5ker } & 355.5 & \multicolumn{2}{|c|}{-166.8} & 317.7 & -165.7 & -2.3 & -162.1 \\
\hline & E3/R5/D7/E11 & \multicolumn{2}{|c|}{ K16 } & E22/D23 & K28 & & A42 \\
\hline & 10 & \multicolumn{2}{|c|}{3} & 1 & 0 & 1 & 2 \\
\hline \multirow{3}{*}{ 5kt0 } & -18.4 & 15.2 & -9.5 & -7.2 & -1.8 & -8.2 & -16.1 \\
\hline & & K16 & V18 & & & L34 & V40 \\
\hline & 15 & 4 & 4 & 0 & 0 & 2 & 0 \\
\hline \multirow{3}{*}{$5 \mathrm{kt1}$} & -10.3 & 11.8 & -8.3 & -9.4 & -7.3 & -7.8 & -11.1 \\
\hline & D1/R5/Y10 & K16 & L17 & & $\mathrm{I} 32$ & L34 & V40 \\
\hline & 12 & 2 & 4 & 2 & 1 & 3 & 0 \\
\hline
\end{tabular}

Table S2. Twelve poses of one drug docked to the 2MXU and the corresponding binding energy

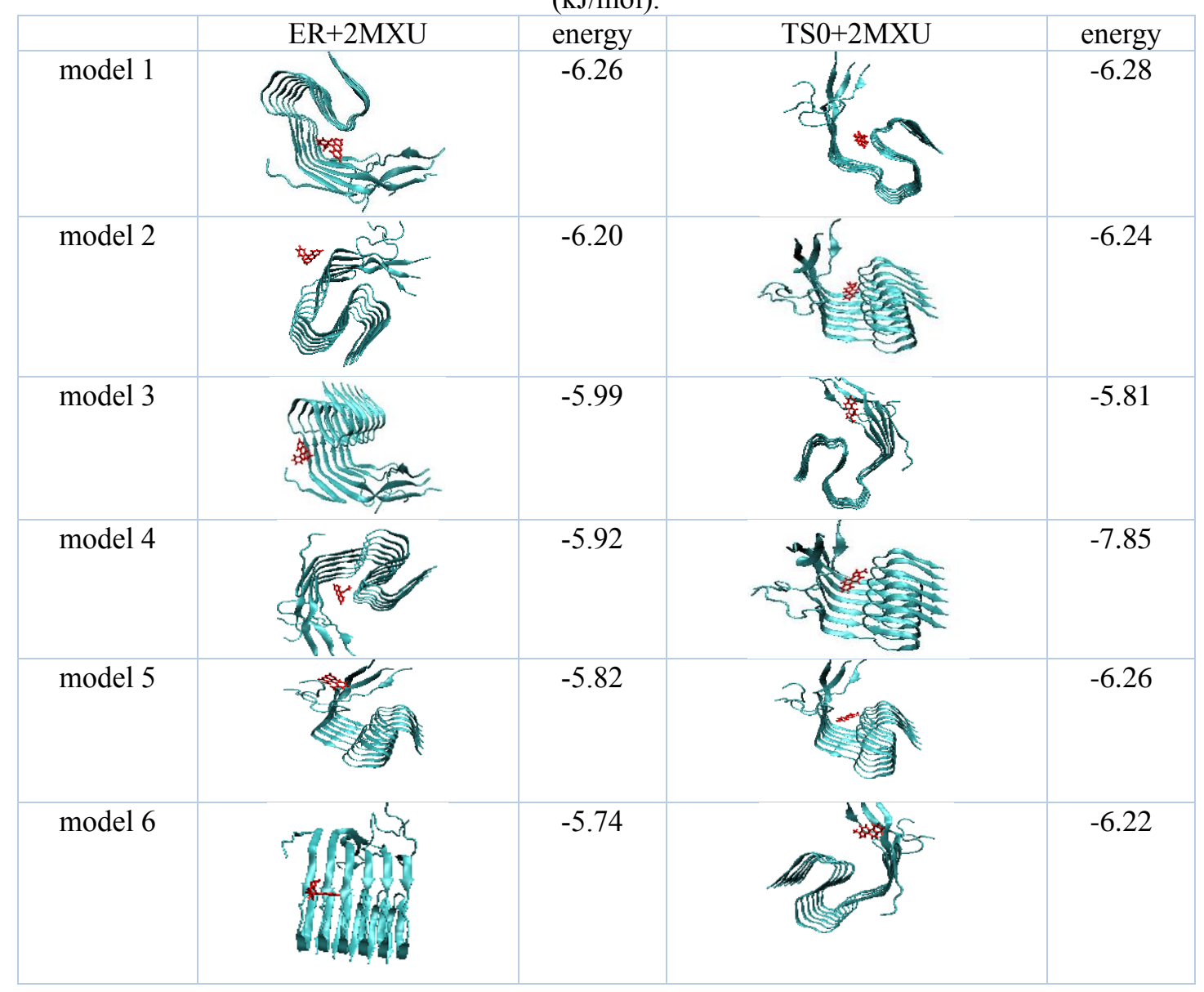




\begin{tabular}{|c|c|c|c|}
\hline model 7 & ( & -5.49 & -6.08 \\
\hline model 8 & & -5.35 & -6.06 \\
\hline model 9 & & -9.05 & -5.19 \\
\hline model 10 & & -8.06 & -6.26 \\
\hline model 11 & 2 & -7.86 & -6.20 \\
\hline model 12 & & -7.14 & -5.99 \\
\hline
\end{tabular}

Table S3. The number of drugs within $10 \AA$ of $2 \mathrm{MXU} / 5 \mathrm{KK} 3$ in each region after docking.

\begin{tabular}{lcccccc}
\hline & $\mathbf{D R}^{\prime \prime \prime \prime}$ & $\boldsymbol{\beta 1} 1^{\prime \prime \prime \prime}$ & $\mathbf{L 1}^{\prime \prime \prime \prime}$ & $\boldsymbol{\beta 2}^{\prime \prime \prime \prime}$ & $\mathbf{L 2}^{\prime \prime \prime \prime}$ & $\boldsymbol{\beta 3}^{\prime \prime \prime \prime}$ \\
\hline 2mer & 3 & 1 & 3 & 2 & 2 & 1 \\
2mt1 & 2 & 6 & 4 & 0 & 0 & 0 \\
2mt0 & 3 & 5 & 4 & 0 & 0 & 0 \\
5ker & 15 & 5 & 0 & 2 & 3 & 2 \\
5kt0 & 12 & 7 & 0 & 3 & 1 & 1 \\
5kt1 & 9 & 5 & 4 & 3 & 1 & 2 \\
\hline
\end{tabular}

Table S4: Binding free energies $\left(\Delta \mathrm{G}_{\mathrm{bind}}\right)$ for $5 \mathrm{KK} 3 / 5 \mathrm{ker} / 5 \mathrm{kt} 0$.

\begin{tabular}{c|c|c}
\hline system & $\Delta \mathrm{G}_{\text {bind }}\left(\mathrm{kcal} \mathrm{mol}^{-1}\right)^{\mathrm{a}}$ & $\Delta \Delta \mathrm{G}_{\text {bind }}\left(\mathrm{kcal} \mathrm{mol}^{-1}\right)$ \\
\hline $5 \mathrm{KK} 3$ & 60.9 & - \\
$5 \mathrm{ker}$ & 48.7 & -12.3 \\
$5 \mathrm{kt0}$ & 50.7 & -10.2
\end{tabular}

${ }^{\text {a }}$ The error associated with energy minima is $\pm 0.3 \mathrm{kcal} \mathrm{mol}^{-1}$ for each system. 
Table S5. Force field parameters of TS0 produced by ATB (Taken as an example).

[ moleculetype ]

$\begin{array}{lll}\text {; Name } & \text { nrexcl } \\ \text { TSO } & 3^{2}\end{array}$

[ atoms ]

; $\mathrm{nr}$ type resnr residue atom cgnr charge mass typeB chargeB

$\begin{array}{llllllll}1 & \text { cc } & 1 & \text { TS0 } & \text { C } & 1 & -0.14060 & 12.000000\end{array}$

$\begin{array}{llllllll}2 & \text { cc } & 1 & \text { TSO } & \text { C1 } & 2 & -0.29490 & 12.000000\end{array}$

$\begin{array}{llllllll}3 & \mathrm{c} & 1 & \mathrm{TS} 0 & \mathrm{C} 2 & 3 & 0.51570 & 12.000000\end{array}$

$\begin{array}{llllllll}4 & \text { o } & 1 & \text { TSO } & \text { O } & 4 & -0.46710 & 16.000000\end{array}$

$\begin{array}{lllllllll}5 & \mathrm{c} & 1 & \mathrm{TS} 0 & \mathrm{C} 3 & 5 & 0.48170 & 12.000000\end{array}$

$\begin{array}{llllllll}6 & \mathrm{o} & 1 & \text { TS0 } & \text { O1 } & 6 & -0.47610 & 16.000000\end{array}$

$\begin{array}{llllllll}7 & \text { ca } & 1 & \text { TS0 } & \text { C4 } & 7 & -0.12160 & 12.000000\end{array}$

$\begin{array}{lllllllll}8 & \text { ca } & 1 & \text { TS0 } & \text { C5 } & 8 & 0.00600 & 12.000000\end{array}$

$\begin{array}{llllllll}9 & \text { ca } & 1 & \text { TS0 } & \text { C6 } & 9 & -0.12000 & 12.000000\end{array}$

10 ha 1 TS0 $\mathrm{H} \quad 10 \quad 0.17300 \quad 1.000000$

$\begin{array}{llllllll}11 & \text { ca } & 1 & \text { TS0 } & \text { C7 } & 11 & -0.12100 & 12.000000\end{array}$

12 ha 1 TS0 H1 $12 \quad 0.14000 \quad 1.000000$

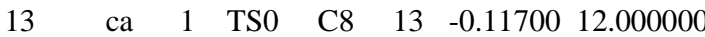

14 ha 1 TS0 $\mathrm{H} 2 \quad 14 \quad 0.13800 \quad 1.000000$

$\begin{array}{llllllll}15 & \text { ca } & 1 & \text { TS0 } & \text { C9 } & 15 & -0.12400 & 12.000000\end{array}$

16 ha 1 TSO H3 $16 \quad 0.13600 \quad 1.000000$

$\begin{array}{llllllll}17 & \text { ca } & 1 & \text { TS0 } & \text { C10 } & 17 & -0.04400 & 12.000000\end{array}$

$\begin{array}{llllllll}18 & \text { ca } & 1 & \text { TS0 } & \text { C11 } & 18 & -0.08400 & 12.000000\end{array}$

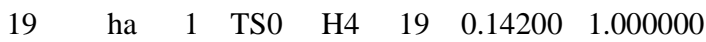

$\begin{array}{llllllll}20 & \text { ca } & 1 & \text { TS0 } & \text { C12 } & 20 & -0.12500 & 12.000000\end{array}$

21 ha 1 TS0 H5 $21 \quad 0.15400 \quad 1.000000$

$\begin{array}{llllllll}22 & \text { ca } & 1 & \text { TS0 } & \text { C13 } & 22 & -0.08850 & 12.000000\end{array}$

$\begin{array}{llllllll}23 & \text { cd } & 1 & \text { TS0 } & \text { C14 } & 23 & 0.34820 & 12.000000\end{array}$

$\begin{array}{llllllll}24 & \text { os } & 1 & \text { TS0 } & \text { O2 } & 24 & -0.33680 & 16.000000\end{array}$

$\begin{array}{llllllll}25 & \text { cd } & 1 & \text { TS0 } & \text { C15 } & 25 & 0.22960 & 12.000000\end{array}$

$\begin{array}{llllllll}26 & \text { h4 } & 1 & \text { TS0 } & \text { H6 } & 26 & 0.06210 & 1.000000\end{array}$

$\begin{array}{llllllll}27 & \text { c3 } & 1 & \text { TS0 } & \text { C16 } & 27 & -0.03580 & 12.000000\end{array}$

$\begin{array}{llllllll}28 & \text { hc } & 1 & \text { TS0 } & \text { H7 } & 28 & 0.05670 & 1.000000\end{array}$

$\begin{array}{llllllll}29 & \text { hc } & 1 & \text { TS0 } & \text { H8 } & 29 & 0.05670 & 1.000000\end{array}$

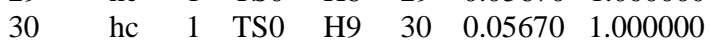

[ bonds ]

; ai aj funct $\mathrm{r} \mathrm{k}$

$9 \quad 10 \quad 1 \quad 1.0870 \mathrm{e}-01 \quad 2.8811 \mathrm{e}+05$

$11 \quad 12 \quad 1 \quad 1.0870 \mathrm{e}-01 \quad 2.8811 \mathrm{e}+05$

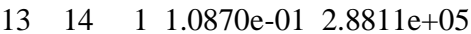

$15 \quad 16 \quad 1 \quad 1.0870 \mathrm{e}-01 \quad 2.8811 \mathrm{e}+05$

$\begin{array}{lllll}18 & 19 \quad 1 & 1.0870 \mathrm{e}-01 & 2.8811 \mathrm{e}+05\end{array}$

$20 \quad 21 \quad 1 \quad 1.0870 \mathrm{e}-01 \quad 2.8811 \mathrm{e}+05$

$25 \quad 26 \quad 1 \quad 1.0830 \mathrm{e}-01 \quad 2.9296 \mathrm{e}+05$

$27 \quad 28 \quad 1 \quad 1.0920 \mathrm{e}-01 \quad 2.8225 \mathrm{e}+05$

$27 \quad 29 \quad 1 \quad 1.0920 \mathrm{e}-01 \quad 2.8225 \mathrm{e}+05$

$27 \quad 30 \quad 1 \quad 1.0920 \mathrm{e}-01 \quad 2.8225 \mathrm{e}+05$

$\begin{array}{lllll}1 & 2 & 1 & 1.4290 \mathrm{e}-01 & 3.5003 \mathrm{e}+05\end{array}$

$125 \quad 1 \quad 1.3710 \mathrm{e}-01 \quad 4.2175 \mathrm{e}+05$

$\begin{array}{lllll}1 & 27 & 1 & 1.4990 \mathrm{e}-01 & 2.8225 \mathrm{e}+05\end{array}$

$23 \quad 1 \quad 1.4620 \mathrm{e}-01 \quad 3.1581 \mathrm{e}+05$

$2 \quad 23 \quad 1 \quad 1.3710 \mathrm{e}-01 \quad 4.2175 \mathrm{e}+05$

$34 \quad 1 \quad 1.2140 \mathrm{e}-01 \quad 5.4225 \mathrm{e}+05$

$\begin{array}{lllll}3 & 5 & 1 & 1.5500 \mathrm{e}-01 & 2.4276 \mathrm{e}+05\end{array}$

$5 \quad 6 \quad 1 \quad 1.2140 \mathrm{e}-01 \quad 5.4225 \mathrm{e}+05$

$\begin{array}{lllllll}5 & 7 & 1 & 1.4870 \mathrm{e}-01 & 2.9263 \mathrm{e}+05\end{array}$

$\begin{array}{lllllll}7 & 8 & 1 & 1.3870 \mathrm{e}-01 & 4.0033 \mathrm{e}+05\end{array}$

$\begin{array}{lllll}7 & 22 & 1 & 1.3870 \mathrm{e}-01 & 4.0033 \mathrm{e}+05\end{array}$

$\begin{array}{llllll}8 & 9 & 1 & 1.3870 \mathrm{e}-01 & 4.0033 \mathrm{e}+05\end{array}$

$\begin{array}{lllllll}8 & 17 & 1 & 1.3870 \mathrm{e}-01 & 4.0033 \mathrm{e}+05\end{array}$

$\begin{array}{llllll}9 & 11 & 1 & 1.3870 \mathrm{e}-01 & 4.0033 \mathrm{e}+05\end{array}$

$11 \quad 13 \quad 1 \quad 1.3870 \mathrm{e}-01 \quad 4.0033 \mathrm{e}+05$ 


$\begin{array}{lllll}13 & 15 & 1 & 1.3870 \mathrm{e}-01 & 4.0033 \mathrm{e}+05 \\ 15 & 17 & 1 & 1.3870 \mathrm{e}-01 & 4.0033 \mathrm{e}+05 \\ 17 & 18 & 1 & 1.3870 \mathrm{e}-01 & 4.0033 \mathrm{e}+05 \\ 18 & 20 & 1 & 1.3870 \mathrm{e}-01 & 4.0033 \mathrm{e}+05 \\ 20 & 22 & 1 & 1.3870 \mathrm{e}-01 & 4.0033 \mathrm{e}+05 \\ 22 & 23 & 1 & 1.4340 \mathrm{e}-01 & 3.4451 \mathrm{e}+05 \\ 23 & 24 & 1 & 1.3700 \mathrm{e}-01 & 3.1472 \mathrm{e}+05 \\ 24 & 25 & 1 & 1.3700 \mathrm{e}-01 & 3.1472 \mathrm{e}+05\end{array}$

[ pairs ]

; ai aj funct

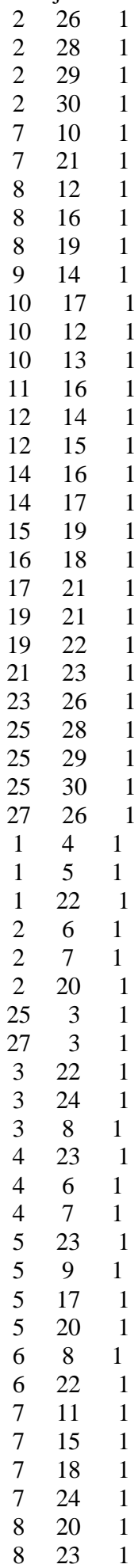




$\begin{array}{ccc}8 & 13 & 1 \\ 9 & 22 & 1 \\ 9 & 15 & 1 \\ 9 & 18 & 1 \\ 11 & 17 & 1 \\ 13 & 18 & 1 \\ 15 & 20 & 1 \\ 17 & 22 & 1 \\ 18 & 23 & 1 \\ 20 & 24 & 1 \\ 22 & 25 & 1 \\ 27 & 23 & 1 \\ 27 & 24 & 1\end{array}$

[ angles ]

; ai aj ak funct theta cth

$\begin{array}{lllllll}1 & 25 & 26 & 1 & 1.2911 \mathrm{e}+02 & 3.9489 \mathrm{e}+02\end{array}$

$\begin{array}{lllllll}1 & 27 & 28 & 1 & 1.1086 \mathrm{e}+02 & 3.9497 \mathrm{e}+02\end{array}$

$\begin{array}{lllllll}1 & 27 & 29 & 1 & 1.1086 \mathrm{e}+02 & 3.9497 \mathrm{e}+02\end{array}$

$\begin{array}{llllllll}1 & 27 & 30 & 1 & 1.1086 \mathrm{e}+02 & 3.9497 \mathrm{e}+02\end{array}$

$\begin{array}{llllllll}8 & 9 & 10 & 1 & 1.2001 \mathrm{e}+02 & 4.0551 \mathrm{e}+02\end{array}$

$\begin{array}{llllll}9 & 11 & 12 & 1 & 1.2001 \mathrm{e}+02 & 4.0551 \mathrm{e}+02\end{array}$

$\begin{array}{llllllll}10 & 9 & 11 & 1 & 1.2001 \mathrm{e}+02 & 4.0551 \mathrm{e}+02\end{array}$

$\begin{array}{llllllll}11 & 13 & 14 & 1 & 1.2001 \mathrm{e}+02 & 4.0551 \mathrm{e}+02\end{array}$

$\begin{array}{llllll}12 & 11 & 13 & 1 & 1.2001 \mathrm{e}+02 & 4.0551 \mathrm{e}+02\end{array}$

$\begin{array}{llllll}13 & 15 & 16 & 1 & 1.2001 \mathrm{e}+02 & 4.0551 \mathrm{e}+02\end{array}$

$\begin{array}{lllllll}14 & 13 & 15 & 1 & 1.2001 \mathrm{e}+02 & 4.0551 \mathrm{e}+02\end{array}$

$\begin{array}{llllll}16 & 15 & 17 & 1 & 1.2001 \mathrm{e}+02 & 4.0551 \mathrm{e}+02\end{array}$

$\begin{array}{llllll}17 & 18 & 19 & 1 & 1.2001 \mathrm{e}+02 & 4.0551 \mathrm{e}+02\end{array}$

$\begin{array}{llllll}18 & 20 & 21 & 1 & 1.2001 \mathrm{e}+02 & 4.0551 \mathrm{e}+02\end{array}$

$\begin{array}{lllllll}19 & 18 & 20 & 1 & 1.2001 \mathrm{e}+02 & 4.0551 \mathrm{e}+02\end{array}$

$\begin{array}{llllll}21 & 20 & 22 & 1 & 1.2001 \mathrm{e}+02 & 4.0551 \mathrm{e}+02\end{array}$

$\begin{array}{lllllll}24 & 25 & 26 & 1 & 1.1189 \mathrm{e}+02 & 4.3740 \mathrm{e}+02\end{array}$

$\begin{array}{lllllll}28 & 27 & 29 & 1 & 1.0835 \mathrm{e}+02 & 3.2995 \mathrm{e}+02\end{array}$

$\begin{array}{lllllll}28 & 27 & 30 & 1 & 1.0835 \mathrm{e}+02 & 3.2995 \mathrm{e}+02\end{array}$

$\begin{array}{lllllll}29 & 27 & 30 & 1 & 1.0835 \mathrm{e}+02 & 3.2995 \mathrm{e}+02\end{array}$

$\begin{array}{llllllllllll}1 & 2 & 3 & 1 & 1.2269 \mathrm{e}+02 & 5.3321 \mathrm{e}+02\end{array}$

$\begin{array}{llllllll}1 & 2 & 23 & 1 & 1.1419 \mathrm{e}+02 & 5.7036 \mathrm{e}+02\end{array}$

$\begin{array}{lllllll}1 & 25 & 24 & 1 & 1.2030 \mathrm{e}+02 & 5.8543 \mathrm{e}+02\end{array}$

$\begin{array}{llllll}2 & 1 & 25 & 1 & 1.1419 \mathrm{e}+02 & 5.7036 \mathrm{e}+02\end{array}$

$\begin{array}{lllllll}2 & 1 & 27 & 1 & 1.1597 \mathrm{e}+02 & 5.4107 \mathrm{e}+02\end{array}$

$\begin{array}{llllll}2 & 3 & 4 & 1 & 1.2571 \mathrm{e}+02 & 5.7664 \mathrm{e}+02\end{array}$

$\begin{array}{lllllll}2 & 3 & 5 & 1 & 1.1167 \mathrm{e}+02 & 5.3572 \mathrm{e}+02\end{array}$

$\begin{array}{llllll}2 & 23 & 22 & 1 & 1.1351 \mathrm{e}+02 & 5.7095 \mathrm{e}+02\end{array}$

$\begin{array}{llllll}2 & 23 & 24 & 1 & 1.2030 \mathrm{e}+02 & 5.8543 \mathrm{e}+02\end{array}$

$\begin{array}{llllllll}3 & 2 & 23 & 1 & 1.2151 \mathrm{e}+02 & 5.4584 \mathrm{e}+02\end{array}$

$\begin{array}{llllll}3 & 5 & 6 & 1 & 1.2099 \mathrm{e}+02 & 5.6199 \mathrm{e}+02\end{array}$

$\begin{array}{lllllll}3 & 5 & 7 & 1 & 1.1834 \mathrm{e}+02 & 5.1656 \mathrm{e}+02\end{array}$

$\begin{array}{lllllll}4 & 3 & 5 & 1 & 1.2099 \mathrm{e}+02 & 5.6199 \mathrm{e}+02\end{array}$

$\begin{array}{llllll}5 & 7 & 8 & 1 & 1.2014 \mathrm{e}+02 & 5.4091 \mathrm{e}+02\end{array}$

$\begin{array}{llllllll}5 & 7 & 22 & 1 & 1.2014 \mathrm{e}+02 & 5.4091 \mathrm{e}+02\end{array}$

$\begin{array}{llllll}6 & 5 & 7 & 1 & 1.2344 \mathrm{e}+02 & 5.7463 \mathrm{e}+02\end{array}$

$\begin{array}{lllllll}7 & 8 & 9 & 1 & 1.1997 \mathrm{e}+02 & 5.6216 \mathrm{e}+02\end{array}$

$\begin{array}{llllll}7 & 8 & 17 & 1 & 1.1997 \mathrm{e}+02 & 5.6216 \mathrm{e}+02\end{array}$

$\begin{array}{llllll}7 & 22 & 20 & 1 & 1.1997 \mathrm{e}+02 & 5.6216 \mathrm{e}+02\end{array}$

$\begin{array}{lllllll}7 & 22 & 23 & 1 & 1.2010 \mathrm{e}+02 & 5.5220 \mathrm{e}+02\end{array}$

$\begin{array}{lllllll}8 & 7 & 22 & 1 & 1.1997 \mathrm{e}+02 & 5.6216 \mathrm{e}+02\end{array}$

$\begin{array}{lllllll}8 & 9 & 11 & 1 & 1.1997 \mathrm{e}+02 & 5.6216 \mathrm{e}+02\end{array}$

$\begin{array}{lllllll}8 & 17 & 15 & 1 & 1.1997 \mathrm{e}+02 & 5.6216 \mathrm{e}+02\end{array}$

$\begin{array}{llllllll}8 & 17 & 18 & 1 & 1.1997 \mathrm{e}+02 & 5.6216 \mathrm{e}+02\end{array}$

$\begin{array}{llllll}9 & 8 & 17 & 1 & 1.1997 \mathrm{e}+02 & 5.6216 \mathrm{e}+02\end{array}$

$\begin{array}{llllll}9 & 11 & 13 & 1 & 1.1997 \mathrm{e}+02 & 5.6216 \mathrm{e}+02\end{array}$

$\begin{array}{lllllll}11 & 13 & 15 & 1 & 1.1997 \mathrm{e}+02 & 5.6216 \mathrm{e}+02\end{array}$

$\begin{array}{llllll}13 & 15 & 17 & 1 & 1.1997 \mathrm{e}+02 & 5.6216 \mathrm{e}+02\end{array}$

$\begin{array}{lllllll}15 & 17 & 18 & 1 & 1.1997 \mathrm{e}+02 & 5.6216 \mathrm{e}+02\end{array}$

$\begin{array}{llllll}17 & 18 & 20 & 1 & 1.1997 \mathrm{e}+02 & 5.6216 \mathrm{e}+02\end{array}$ 
$\begin{array}{lllllll}18 & 20 & 22 & 1 & 1.1997 \mathrm{e}+02 & 5.6216 \mathrm{e}+02\end{array}$

$\begin{array}{llllll}20 & 22 & 23 & 1 & 1.2010 \mathrm{e}+02 & 5.5220 \mathrm{e}+02\end{array}$

$\begin{array}{lllllll}22 & 23 & 24 & 1 & 1.1445 \mathrm{e}+02 & 5.8609 \mathrm{e}+02\end{array}$

$\begin{array}{llllll}23 & 24 & 25 & 1 & 1.0645 \mathrm{e}+02 & 5.6358 \mathrm{e}+02\end{array}$

$\begin{array}{llllll}25 & 1 & 27 & 1 & 1.1945 \mathrm{e}+02 & 5.4233 \mathrm{e}+02\end{array}$

[ dihedrals ]

;i j k 1 func

$\begin{array}{lllllll}2 & 1 & 25 & 26 & 3 & 33 & \text { C5 }\end{array}$

$\begin{array}{llllll}2 & 1 & 27 & 28 & 3 & 0.0000\end{array}$

$\begin{array}{llllll}2 & 1 & 27 & 29 & 3 & 0.00000\end{array}$

$\begin{array}{llllll}2 & 1 & 27 & 30 & 3 & 0.00000\end{array}$

$\begin{array}{llllll}7 & 8 & 9 & 10 & 3 & 30.33400\end{array}$

$\begin{array}{llllll}7 & 22 & 20 & 21 & 3 & 30.33400\end{array}$

$\begin{array}{llllll}8 & 9 & 11 & 12 & 3 & 30.33400\end{array}$

$\begin{array}{llllll}8 & 17 & 15 & 16 & 3 & 30.33400\end{array}$

$\begin{array}{llllll}8 & 17 & 18 & 19 & 3 & 30.33400\end{array}$

$\begin{array}{llllll}9 & 11 & 13 & 14 & 3 & 30.33400\end{array}$

$\begin{array}{llllll}10 & 9 & 8 & 17 & 3 & 30.33400\end{array}$

$\begin{array}{llllll}10 & 9 & 11 & 12 & 3 & 30.33400\end{array}$

$\begin{array}{llllll}10 & 9 & 11 & 13 & 3 & 30.33400\end{array}$

$\begin{array}{llllll}11 & 13 & 15 & 16 & 3 & 30.33400\end{array}$

$\begin{array}{llllll}12 & 11 & 13 & 14 & 3 & 30.33400\end{array}$

$\begin{array}{llllll}12 & 11 & 13 & 15 & 3 & 30.33400\end{array}$

$\begin{array}{llllll}14 & 13 & 15 & 16 & 3 & 30.33400\end{array}$

$\begin{array}{llllll}14 & 13 & 15 & 17 & 3 & 30.33400\end{array}$

$\begin{array}{llllll}15 & 17 & 18 & 19 & 3 & 30.33400\end{array}$

$\begin{array}{llllll}16 & 15 & 17 & 18 & 3 & 30.33400\end{array}$

$\begin{array}{llllll}17 & 18 & 20 & 21 & 3 & 30.33400\end{array}$

$\begin{array}{llllll}19 & 18 & 20 & 21 & 3 & 30.33400\end{array}$

$\begin{array}{llllll}19 & 18 & 20 & 22 & 3 & 30.33400\end{array}$

$\begin{array}{llllll}21 & 20 & 22 & 23 & 3 & 30.33400\end{array}$

$\begin{array}{llllll}23 & 24 & 25 & 26 & 3 & 8.78640\end{array}$

$\begin{array}{llllll}25 & 1 & 27 & 28 & 3 & 0.00000\end{array}$

$\begin{array}{llllll}25 & 1 & 27 & 29 & 3 & 0.00000\end{array}$

$\begin{array}{llllll}25 & 1 & 27 & 30 & 3 & 0.00000\end{array}$

$\begin{array}{llllll}27 & 1 & 25 & 26 & 3 & 33.47200\end{array}$

$\begin{array}{llllll}8 & 11 & 9 & 10 & 3 & 9.20480\end{array}$

$\begin{array}{llllll}9 & 13 & 11 & 12 & 3 & 9.20480\end{array}$

$\begin{array}{llllll}11 & 15 & 13 & 14 & 3 & 9.20480\end{array}$

$\begin{array}{llllll}13 & 17 & 15 & 16 & 3 & 9.20480\end{array}$

$\begin{array}{llllll}17 & 20 & 18 & 19 & 3 & 9.20480\end{array}$

$\begin{array}{llllll}18 & 22 & 20 & 21 & 3 & 9.20480\end{array}$

$\begin{array}{llllll}1 & 26 & 25 & 24 & 3 & 9.20480\end{array}$

$\begin{array}{lllllll}1 & 2 & 3 & 4 & 3 & 24.05800\end{array}$

$\begin{array}{llllll}1 & 2 & 3 & 5 & 3 & 24.05800\end{array}$

$\begin{array}{llllll}1 & 2 & 23 & 22 & 3 & 33.47200\end{array}$

$\begin{array}{llllll}1 & 2 & 23 & 24 & 3 & 33.47200\end{array}$

$\begin{array}{llllll}1 & 25 & 24 & 23 & 3 & 8.78640\end{array}$

$\begin{array}{llllll}2 & 1 & 25 & 24 & 3 & 33.47200\end{array}$

$\begin{array}{llllll}2 & 3 & 5 & 6 & 3 & 2.51040\end{array}$

$\begin{array}{lllll}0.00000 & -33.47200 & 0.00000 & 0.00000 & 0.00000\end{array}$

$\begin{array}{llllll}0.00000 & 0.00000 & 0.00000 & 0.00000 & 0.00000 ;\end{array}$

$\begin{array}{lllll}0.00000 & 0.00000 & 0.00000 & 0.00000 & 0.00000 ;\end{array}$

$\begin{array}{llllll}0.00000 & 0.00000 & 0.00000 & 0.00000 & 0.00000\end{array}$;

$\begin{array}{llllll}0.00000 & -30.33400 & 0.00000 & 0.00000 & 0.00000 \text {; }\end{array}$

$\begin{array}{lllll}0.00000 & -30.33400 & 0.00000 & 0.00000 & 0.00000\end{array}$

$\begin{array}{lllll}0.00000 & -30.33400 & 0.00000 & 0.00000 & 0.00000\end{array}$

$\begin{array}{lllll}0.00000 & -30.33400 & 0.00000 & 0.00000 & 0.00000\end{array}$

$\begin{array}{lllll}0.00000 & -30.33400 & 0.00000 & 0.00000 & 0.00000\end{array}$

$\begin{array}{lllll}0.00000 & -30.33400 & 0.00000 & 0.00000 & 0.00000\end{array}$

$\begin{array}{lllll}0.00000 & -30.33400 & 0.00000 & 0.00000 & 0.00000\end{array}$

$\begin{array}{llllll}0.00000 & -30.33400 & 0.00000 & 0.00000 & 0.00000\end{array}$

$\begin{array}{llllll}0.00000 & -30.33400 & 0.00000 & 0.00000 & 0.00000\end{array}$

$\begin{array}{llllll}0.00000 & -30.33400 & 0.00000 & 0.00000 & 0.00000\end{array}$

$\begin{array}{lllll}0.00000 & -30.33400 & 0.00000 & 0.00000 & 0.00000\end{array}$

$\begin{array}{llllll}0.00000 & -30.33400 & 0.00000 & 0.00000 & 0.00000\end{array}$

$\begin{array}{lllll}0.00000 & -30.33400 & 0.00000 & 0.00000 & 0.00000\end{array}$

$\begin{array}{llllll}0.00000 & -30.33400 & 0.00000 & 0.00000 & 0.00000\end{array}$

$\begin{array}{lllll}0.00000 & -30.33400 & 0.00000 & 0.00000 & 0.00000\end{array}$

$\begin{array}{lllll}0.00000 & -30.33400 & 0.00000 & 0.00000 & 0.00000\end{array}$

$\begin{array}{lllll}0.00000 & -30.33400 & 0.00000 & 0.00000 & 0.00000\end{array}$

$\begin{array}{lllll}0.00000 & -30.33400 & 0.00000 & 0.00000 & 0.00000\end{array}$

$\begin{array}{lllll}0.00000 & -30.33400 & 0.00000 & 0.00000 & 0.00000\end{array}$

$\begin{array}{lllll}0.00000 & -30.33400 & 0.00000 & 0.00000 & 0.00000\end{array}$

$\begin{array}{lllll}0.00000 & -8.78640 & 0.00000 & 0.00000 & 0.00000\end{array}$

$\begin{array}{lllll}0.00000 & 0.00000 & 0.00000 & 0.00000 & 0.00000 ;\end{array}$

$\begin{array}{llllll}0.00000 & 0.00000 & 0.00000 & 0.00000 & 0.00000 ;\end{array}$

$\begin{array}{llllll}0.00000 & 0.00000 & 0.00000 & 0.00000 & 0.00000 ;\end{array}$

$\begin{array}{lllll}0.00000 & -33.47200 & 0.00000 & 0.00000 & 0.00000\end{array}$

$\begin{array}{lllll}0.00000 & -9.20480 & 0.00000 & 0.00000 & 0.00000 ;\end{array}$

$\begin{array}{llllll}0.00000 & -9.20480 & 0.00000 & 0.00000 & 0.00000 ;\end{array}$

$\begin{array}{llllll}0.00000 & -9.20480 & 0.00000 & 0.00000 & 0.00000\end{array}$

$\begin{array}{lllll}0.00000 & -9.20480 & 0.00000 & 0.00000 & 0.00000\end{array}$

$\begin{array}{lllll}0.00000 & -9.20480 & 0.00000 & 0.00000 & 0.00000\end{array}$

$\begin{array}{lllll}0.00000 & -9.20480 & 0.00000 & 0.00000 & 0.00000\end{array}$

$\begin{array}{lllll}0.00000 & -9.20480 & 0.00000 & 0.00000 & 0.00000\end{array}$

$\begin{array}{llllll}0.00000 & -24.05800 & 0.00000 & 0.00000 & 0.00000 ;\end{array}$

$\begin{array}{lllll}0.00000 & -24.05800 & 0.00000 & 0.00000 & 0.00000 ;\end{array}$

$\begin{array}{lllll}0.00000 & -33.47200 & 0.00000 & 0.00000 & 0.00000\end{array}$

$\begin{array}{lllll}0.00000 & -33.47200 & 0.00000 & 0.00000 & 0.00000\end{array}$

$\begin{array}{lllll}0.00000 & -8.78640 & 0.00000 & 0.00000 & 0.00000\end{array}$

$\begin{array}{llllllllllll}2 & 3 & 5 & 7 & 3 & 2.51040 & 0.00000 & -2.51040 & 0.00000 & 0.00000 & 0.00000 & ;\end{array}$

$\begin{array}{lllllllllll}2 & 23 & 22 & 7 & 3 & 21.33840 & 0.00000 & -21.33840 & 0.00000 & 0.00000 & 0.00000\end{array}$

$\begin{array}{llllllllllll}2 & 23 & 22 & 20 & 3 & 21.33840 & 0.00000 & -21.33840 & 0.00000 & 0.00000 & 0.00000\end{array}$

$\begin{array}{lllllllllll}2 & 23 & 24 & 25 & 3 & 8.78640 & 0.00000 & -8.78640 & 0.00000 & 0.00000 & 0.00000 \text {; }\end{array}$

$\begin{array}{lllllllllll}25 & 1 & 2 & 3 & 3 & 33.47200 & 0.00000 & -33.47200 & 0.00000 & 0.00000 & 0.00000 ;\end{array}$

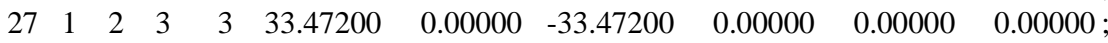

$\begin{array}{lllllllllll}3 & 2 & 23 & 22 & 3 & 33.47200 & 0.00000 & -33.47200 & 0.00000 & 0.00000 & 0.00000\end{array}$

$\begin{array}{llllllllllll}3 & 2 & 23 & 24 & 3 & 33.47200 & 0.00000 & -33.47200 & 0.00000 & 0.00000 & 0.00000\end{array}$

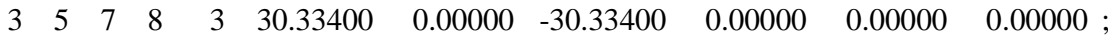

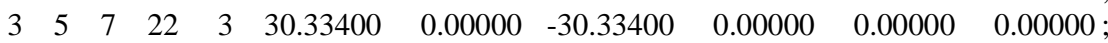

$\begin{array}{llllllllllll}4 & 3 & 2 & 23 & 3 & 24.05800 & 0.00000 & -24.05800 & 0.00000 & 0.00000 & 0.00000 ;\end{array}$

$\begin{array}{lllllllllll}4 & 3 & 5 & 6 & 3 & 2.51040 & 0.00000 & -2.51040 & 0.00000 & 0.00000 & 0.00000\end{array}$;

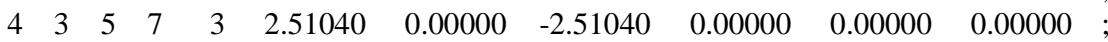

$\begin{array}{llllllllllll}5 & 3 & 2 & 23 & 3 & 24.05800 & 0.00000 & -24.05800 & 0.00000 & 0.00000 & 0.00000 ;\end{array}$

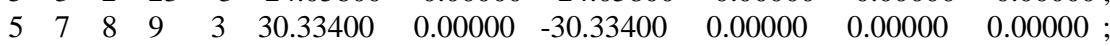




\begin{tabular}{|c|c|c|c|c|c|c|c|c|c|c|}
\hline & 7 & 8 & 17 & 3 & & & & & & \\
\hline & 7 & 22 & 20 & 3 & & 000 & & & & \\
\hline & 7 & 22 & 23 & 3 & 400 & 0.00000 & 3400 & 0.00000 & 0000 & \\
\hline & 5 & 7 & 8 & 3 & 30.33400 & 0.00000 & $-30 .$. & 0.00000 & 0.00000 & \\
\hline & 5 & 7 & 22 & 3 & 30. & 00000 & & 0.00000 & & \\
\hline & 8 & 9 & 11 & 3 & & 00000 & & 0.00000 & & \\
\hline & 8 & 17 & 15 & 3 & 30 . & 000 & & & 00 & \\
\hline & 8 & 17 & 18 & 3 & 30 . & 000 & & & 00 & \\
\hline & 22 & 20 & 18 & 3 & & 000 & & 000 & 000 & \\
\hline & 22 & 23 & 24 & 3 & & & & & & \\
\hline & 7 & 22 & 20 & 3 & & & & & & \\
\hline & 7 & 22 & 23 & 3 & & & & & & \\
\hline & 9 & 11 & 13 & 3 & & & & & & \\
\hline & 17 & 15 & 13 & 3 & & & & & & \\
\hline & 17 & 18 & 20 & 3 & & & & & & \\
\hline & 8 & 7 & 22 & 3 & & & & & & \\
\hline & 8 & 17 & 15 & 3 & & & & & & \\
\hline & 8 & 17 & 18 & 3 & & & & & & \\
\hline & 11 & 13 & 15 & 3 & & & & & & \\
\hline & 9 & 8 & 17 & 3 & & & & & & \\
\hline & 13 & 15 & $5 \quad 17$ & 3 & & & & & & \\
\hline & 15 & 17 & $7 \quad 18$ & 3 & & & & & & \\
\hline & 17 & 18 & 20 & 3 & & & & & & \\
\hline & 8 & 7 & 22 & 3 & 30 . & & & & & \\
\hline & 18 & 20 & 22 & 3 & & & & & & \\
\hline & 20 & 22 & 23 & 3 & & & & & & \\
\hline & 22 & 23 & 324 & 3 & & & & & & \\
\hline & 23 & 24 & 425 & 3 & & & & & & \\
\hline & 1 & 2 & 23 & 3 & & & & & & \\
\hline & 1 & 2 & 23 & 3 & & & & & & \\
\hline & 1 & 25 & 24 & 3 & & & & 000 & & \\
\hline & 1 & 2 & 27 & 3 & & & & .00000 & & \\
\hline & 1 & 2 & 23 & 3 & & & -9.2 & 0 & & \\
\hline & 23 & 32 & 4 & 3 & & & & & & \\
\hline & 7 & 5 & 6 & 3 & & & & & & \\
\hline & 8 & 72 & 22 & 3 & & & & & & \\
\hline & 9 & 8 & 17 & 3 & & & & & & \\
\hline & 15 & 17 & 18 & 3 & & & & & & \\
\hline & 20 & 22 & 23 & 3 & & & & & & \\
\hline & 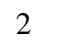 & 23 & 24 & 3 & 0010 & 0.00000 & -9.20480 & 0.00000 & 0.00000 & \\
\hline
\end{tabular}

